Article

\title{
Certain Concepts of Bipolar Fuzzy Directed Hypergraphs
}

\author{
Muhammad Akram * and Anam Luqman \\ Department of Mathematics, University of the Punjab, New Campus, Lahore 54590, Pakistan; \\ anamluqman7@yahoo.com \\ * Correspondence: m.akram@pucit.edu.pk; Tel.: +92-42-9923-1241
}

Academic Editor: Lokenath Debnath

Received: 15 December 2016; Accepted: 22 February 2017; Published: 4 March 2017

\begin{abstract}
A hypergraph is the most developed tool for modeling various practical problems in different fields, including computer sciences, biological sciences, social networks and psychology. Sometimes, given data in a network model are based on bipolar information rather than one sided. To deal with such types of problems, we use mathematical models that are based on bipolar fuzzy (BF) sets. In this research paper, we introduce the concept of BF directed hypergraphs. We describe certain operations on BF directed hypergraphs, including addition, multiplication, vertex-wise multiplication and structural subtraction. We introduce the concept of $B=\left(m^{+}, m^{-}\right)$-tempered $\mathrm{BF}$ directed hypergraphs and investigate some of their properties. We also present an algorithm to compute the minimum arc length of a BF directed hyperpath.
\end{abstract}

Keywords: bipolar fuzzy (BF) directed hypergraphs; B-tempered BF directed hypergraphs; BF shortest hyperpath

\section{Introduction}

Graph theory has become a powerful conceptual framework for the modeling and solution of combinatorial problems that arise in various areas, including mathematics, computer science and engineering. Graphs are only useful for modeling of the pairwise communication. However, many times (for example, in statistical physics and effective theories), one works with such interactions that are based on more than two particles. To deal with such kinds of interactions, we use a hyperedge, as it contains more than two vertices. Hypergraphs [1], a generalization of graphs, have many properties that are the basis of different techniques that are used in modern mathematics. Hypergraphs are stated as the extended form of ordinary graphs in the way that they contain a finite collection of points and a set of hyperarcs defined as a subset of vertices. The applicability of graph theory has widened by the generalization of undirected graphs, called undirected hypergraphs, which have been proven to be more useful as mathematical modeling tools. In real-world applications, hypergraph techniques appear very useful in many places, including declustering problems, which are important to increase the performance of parallel databases [2]. Hypergraphs can be demonstrated as a useful engine (or tool) to model concepts and systems in different fields of discrete mathematics. There are different types of hypergraphs that have been broadly utilized in computer science as a suitable mathematical model. There are many complex phenomena and concepts in many areas, including rewriting systems, problem solving, databases and logic programming, which can be represented using hypergraphs [3]. The most used hypergraphs in computer science are undirected hypergraphs [4]. Directed hypergraphs are used to solve and model certain problems arising in deductive databases and in model checking.

There are many complicated phenomena in science and technology in which available information is not accurate. For such types of problems, we use mathematical models that contain elements of uncertainty. These mathematical models are based on fuzzy set theory. The idea of fuzzy sets was given 
by Zadeh [5]. Fuzzy set theory has many applications in many disciplines, including management sciences, decision theory and robotics. Fuzzy sets have been used successfully in problems that involve approximate reasoning. Zhang [6] gave the idea of bipolar fuzzy (BF) sets. BFs generalize the fuzzy sets whose degree of membership ranges over $[-1,1]$. There are many problems in which it is necessary to utilize bipolar information. In BFs, there are two types of information, namely positive and negative. Positive information deals with the possibility that an element satisfies some property, whereas negative information deals with the element which satisfies some counter property. In recent years, this domain has motivated new research in several fields. For instance we suppose that we have to determine the location of something in the space, we use positive information to express the set of points that are possible, and the set of places that are impossible is taken as negative information.

In 1973, Kaufmann [7] gave the concept of fuzzy graphs based on Zadeh's fuzzy relations [8]. Rosenfeld [9] described the structure of fuzzy graphs. Later on, some remarks on fuzzy graphs were given by Bhattacharya [10]. In 1994, Mordeson and Chang-Shyh [11] defined some operations on fuzzy graphs. Kaufmann [7] presented the idea of fuzzy hypergraphs. Mordeson and Nair presented a valuable contribution on fuzzy graphs as well as fuzzy hypergraphs in [12]. Interval-valued fuzzy hypergraphs were introduced by Chen [13]. Lee Kwang and K.m Lee studied the fuzzy hypergraphs using fuzzy partition in [14]. Intuitionistic fuzzy directed hypergraphs were defined by Parvathi and Thilagavathi in 2013 [15]. Rangasamy et al. [16] proposed a method for finding the shortest hyperpath in an intuitionistic fuzzy weighted hypergraph. Further, certain types of intuitionistic fuzzy directed hypergraphs were discussed by Myithili et al. in [17]. BF graphs were first defined by Akram in [18]. In 2012, Akram and Dudek discussed the regularity of BF graphs [19]. Novel applications of bipolar fuzzy graphs were discussed by Akram ans Waseem in [20]. Sarwar and Akram discussed the novel concepts of bipolar fuzzy competition graphs in [21]. In 2011, BF hypergraphs were studied by Samanta and Pal [22]. In 2013, Akram et al. [23] discussed the properties of BF hypergraphs.

This paper is organized as follows: In Section 2, the concepts of BF hypergraphs, $\mathrm{BF}$ directed hypergraphs and hyperpath are described. Some certain operations on BF directed hypergraphs, including addition, multiplication, vertex-wise multiplication and structural subtraction, are introduced. The concepts of simple, elementary, support simple and sectionally elementary $\mathrm{BF}$ directed hypergraphs are introduced. This section also deals with $B$-tempered BF directed hypergraphs. In Section 3, we provide an algorithm to compute minimum arc length in a BF directed hypernetwork. The shortest BF directed hyperpath is calculated using the score-based method. In the last section, we conclude with our results. For other notations, terminologies and applications not mentioned in the paper, the readers are referred to [24-26]. Throughout this paper, the following notations given in Table 1 will be used:

Table 1. Notations. BF: bipolar fuzzy.

\begin{tabular}{cc}
\hline Notations & Description \\
\hline$B=\left\langle m^{+}, m^{-}\right\rangle$ & BF set \\
$G=(T, U)$ & BF directed hypergraph having vertex set $T$ and edge set $U$ \\
{$[T, U]$} & Index matrix of $G$ \\
$\mathbf{0}$ & $\langle 0,0\rangle$ \\
$\tilde{B}$ & Triangular BF number \\
$S(\tilde{B})$ & Score of BF number $\tilde{B}$ \\
$A c c(\tilde{B})$ & Accuracy of BF number $\tilde{B}$ \\
$h(G)$ & Height of hypergraph $G$ \\
$F S(G)$ & Fundamental sequence of $G$ \\
$c(G)$ & Core set of $G$ \\
$G\left(\mu_{i}, v_{i}\right)$ & $\left(\mu_{i}, v_{i}\right)$-level BF hypergraph \\
\hline
\end{tabular}




\section{Bipolar Fuzzy Directed Hypergraphs}

Definition 1. [4] A directed hypergraph is a hypergraph with directed hyperedges. A directed hyperedge or hyperarc is an ordered pair $E=(X, Y)$ of (possibly empty) disjoint subsets of vertices. $X$ is the tail of $E$, while $Y$ is its head.

Definition 2. [23] A BF hypergraph is an ordered pair $H=(N, E)$, where:

(1) $N$ is a finite collection of points,

(2) $E=\left\{E_{1}, E_{2}, E_{3}, \ldots, E_{k}\right\}$ is a finite collection of nontrivial BF subsets of $N$,

(3) (i) $E_{j}=\left\{\left(v_{i}, m_{j}^{+}\left(v_{i}\right), m_{j}^{-}\left(v_{i}\right)\right) \mid v_{i} \in N\right\}$,

(ii) $E_{j} \subseteq N \times N$, where $m_{i j}^{+}: N \times N \rightarrow[0,1]$ and $m_{i j}^{-}: N \times N \rightarrow[-1,0]$ are such that:

$$
m_{i j}^{+} \leq \min \left\{m_{i}^{+}, m_{j}^{+}\right\}, m_{i j}^{-} \leq \max \left\{m_{i}^{-}, m_{j}^{-}\right\},
$$

(4) $E_{j} \neq \phi, j=1,2,3, \ldots, k$,

(5) $\quad N=\bigcup_{j} \operatorname{supp}\left(E_{j}\right), j=1,2,3, \ldots, k$.

Here, the edges $E_{j}$ are BF sets. $m_{j}^{+}\left(v_{i}\right)$ and $m_{j}^{-}\left(v_{i}\right)$ are positive and negative membership values of vertex $v_{i}$ to edge $E_{j}$, respectively.

If $m_{i j}^{+}=m_{i j}^{-}=0$, it indicates the non-existence of the edge between $x_{i}$ and $x_{j}$; it is indexed by $\langle 0,0\rangle=\mathbf{0}$. Otherwise, there exists an edge.

We now define the BF directed hypergraph.

Definition 3. A BF directed hyperarc (hyperedge) $a \in U$ is a pair $(t(a), h(a))$, where $t(a) \subset U, t(a) \neq \phi$ is its tail and $h(a) \in U-t(a)$ is called its head. A source vertex $s$ is defined as a vertex in $G$ if $h(a) \neq s$, for each $a \in U$. A destination vertex $d$ is defined as a vertex if $t(a) \neq d$, for every $a \in U$.

A BF directed hypergraph $G$ is a pair $(T, U)$, where $T$ is a finite set of points and $U$ is a set of $B F$ directed hyperarcs.

Definition 4. A BF directed hyperedge (or hyperarc) is defined as an ordered pair $U=(u, v)$, where $u$ and $v$ are disjoint subsets of nodes. $u$ is taken as the tail of $U$ and $v$ is called its head. $t(U)$ and $h(U)$ are used to denote the tail and head of the BF directed hyperarc, respectively.

Definition 5. A backward BF directed hyperarc or b-arc is defined as a hyperarc $U=(t(U), h(U))$, with $|h(U)|=1$. A forward BF hyperarc or f-arc is a hyperarc $U=(t(U), h(U))$, with $|t(U)|=1$.

$A B F$ directed hypergraph is called $a b-B F$ directed hypergraph if its hyperarcs are $b$-arcs. $A$ BF directed hypergraph is said to be a $f$-BF directed hypergraph if its hyperarcs are f-arcs. A backward-forward (bf)-graph (or bf-bipolar fuzzy directed hypergraph) is a BF directed hypergraph whose hyperarcs are either $b$-arcs or $f$-arcs.

Definition 6. A path between nodes s and d in a BF directed hypergraph $G$ is an alternating sequence of distinct vertices and BF hyperedges $s=t_{0}, e_{1}, t_{1}, e_{2}, \ldots, e_{k}=d$, such that $t_{i-1}, t_{i} \in e_{i}$, for all $i=1,2,3, \ldots, k$.

Example 1. A BF directed hypergraph and a hyperpath between two nodes $s$ and $d$ is shown in Figure 1 (generated with LaTeXDraw 2.0.8 Mon17 October 2016 12:01:25 PDT). 


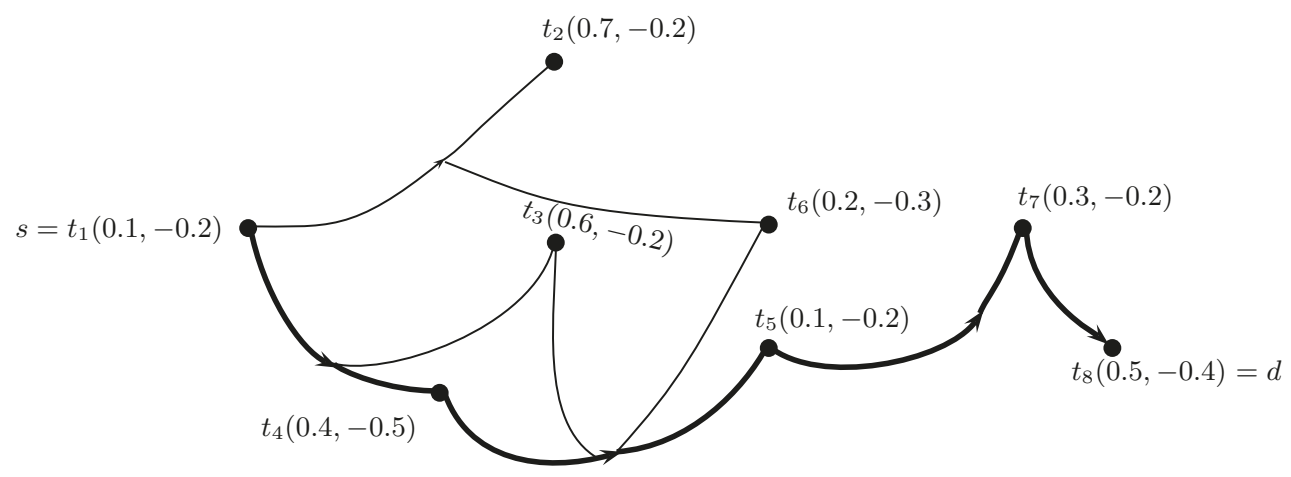

Figure 1. A path from source $s$ to destination node $d$ in a BF set directed hypergraph.

The path is drawn as a thick line.

Definition 7. [4] The incidence matrix representation of a directed hypergraph $N=(V, A)$ is given as a matrix $\left[b_{i j}\right]$ of order $n \times m$, defined as follows:

$$
b_{i j}= \begin{cases}-1, & \text { if } \quad e_{i} \in t\left(A_{j}\right) \\ 1, & \text { if } \quad e_{i} \in h\left(A_{j}\right) \\ 0, & \text { otherwise }\end{cases}
$$

Definition 8. The incidence matrix of a BF directed hypergraph $G=(T, U)$ is characterized by an $n \times m$ matrix $\left[a_{i j}\right]$ as follows:

$$
a_{i j}= \begin{cases}\left\langle m_{j}^{+}\left(n_{i}\right), m_{j}^{-}\left(n_{i}\right)\right\rangle, & \text { if } n_{i} \in U_{j}, \\ \mathbf{0}, & \text { otherwise. }\end{cases}
$$

Definition 9. Let $G=(T, U)$ be a BF directed hypergraph. The height $h(G)$ of $G$ is defined as:

$$
h(G)=\left\{\max \left(U_{i}\right), \min \left(U_{j}\right): U_{i}, U_{j} \in U\right\},
$$

where $U_{i}=\max \left(m_{i j}^{+}\right)$and $U_{j}=\min \left(m_{i j}^{-}\right), m_{i j}^{+}$is taken as the positive membership value and $m_{i j}^{-}$indicates the negative membership value of vertex $i$ to hyperedge $j$.

Definition 10. A BF directed hypergraph $G=(T, U)$ is simple if there are no repeated BF hyperedges in $U$, and if $U_{k}, U_{j} \in U$ and $U_{k} \subseteq U_{j}$, then $U_{k}=U_{j}$, for each $k$ and $j$.

Definition 11. A BF directed hypergraph $G=(T, U)$ is called support simple if whenever $U_{i}, U_{j} \in U$, $U_{i} \subseteq U_{j}$ and $\operatorname{supp}\left(U_{i}\right)=\operatorname{supp}\left(U_{j}\right)$, then $U_{i}=U_{j}$, for all $i$ and $j$. Then, the hyperedges $U_{i}$ and $U_{j}$ are called supporting edges.

Definition 12. A BF directed hypergraph is named elementary if $m_{i j}^{+}: \rightarrow[0,1]$ and $m_{i j}^{-}: \rightarrow[-1,0]$ are constant functions. If $\left|\operatorname{supp}\left(m_{i j}^{+}, m_{i j}^{-}\right)\right|=1$, then it is characterized as a spike; that is, a BF subset with singleton support.

Theorem 1. The BF directed hyperedges of a BF directed hypergraph are elementary.

Example 2. Consider a BF directed hypergraph $G=(T, U)$, such that $T=\left\{t_{1}, t_{2}, t_{3}, t_{4}\right\}, U=$ $\left\{U_{1}, U_{2}, U_{3}, U_{4}\right\}$. The corresponding incidence matrix is given in Table 2. 
Table 2. Elementary BF directed hypergraph.

\begin{tabular}{ccccc}
\hline $\boldsymbol{I}$ & $\boldsymbol{U}_{\mathbf{1}}$ & $\boldsymbol{U}_{\mathbf{2}}$ & $\boldsymbol{U}_{\mathbf{3}}$ & $\boldsymbol{U}_{\mathbf{4}}$ \\
\hline$t_{1}$ & $\langle 0.2,-0.3\rangle$ & $\langle 0.5,-0.2\rangle$ & $\mathbf{0}$ & $\langle 0.3,-0.4\rangle$ \\
$t_{2}$ & $\mathbf{0}$ & $\langle 0.5,-0.2\rangle$ & $\langle 0.5,-0.2\rangle$ & $\mathbf{0}$ \\
$t_{3}$ & $\langle 0.2,-0.3\rangle$ & $\mathbf{0}$ & $\langle 0.5,-0.2\rangle$ & $\langle 0.3,-0.4\rangle$ \\
$t_{4}$ & $\mathbf{0}$ & $\mathbf{0}$ & $\langle 0.5,-0.2\rangle$ & $\langle 0.3,-0.4\rangle$ \\
\hline
\end{tabular}

The corresponding elementary BF directed hypergraph is shown in Figure 2.

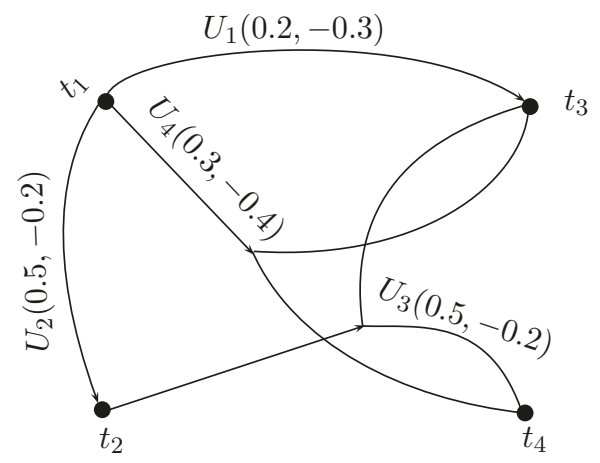

Figure 2. Elementary BF directed hypergraph.

Definition 13. Let $G=(T, U)$ be a BF directed hypergraph. Suppose that $\mu \in[0,1]$ and $v \in[-1,0]$. The $(\mu, v)$-level is defined as $U_{(\mu, v)}=\left\{v \in T \mid m^{+}(v) \geq \mu\right.$ or $\left.m^{-}(v) \leq v\right\}$. The crisp directed hypergraph $\left.G_{(} \mu, v\right)=\left(T_{(\mu, v)}, U_{(\mu, v)}\right)$, such that:

- $U_{(\mu, v)}=\left\{v \in T \mid m^{+}(v) \geq \mu \quad\right.$ or $\left.m^{-}(v) \leq v\right\}$,

- $T_{(\mu, v)}=\bigcup_{U} U_{(\mu, v)}$,

is called the $(\mu, v)$-level hypergraph of $G$.

Definition 14. Let $G=(T, U)$ be a BF directed hypergraph and $G_{\mu_{i}, v_{i}}=\left(T_{\mu_{i}, v_{i}}, U_{\mu_{i}, v_{i}}\right)$ be the $\left(\mu_{i}, v_{i}\right)$-level directed hypergraphs of $G$. The sequence $\left\{\left(\mu_{1}, v_{1}\right),\left(\mu_{2}, v_{2}\right), \ldots,\left(\mu_{n}, v_{n}\right)\right\}$ of real numbers, where $0<\mu_{1}<\mu_{2}<\ldots<\mu_{n}$ and $0>v_{1}>v_{2}>\ldots>v_{n},\left(\mu_{n}, v_{n}\right)=h(G)$, such that the following properties:

(i) if $\left(\mu_{i-1}, v_{i-1}\right)<(\alpha, \beta) \leq\left(\mu_{i}, v_{i}\right)$, then $U_{(\alpha, \beta)}=U_{\left(\mu_{i}, v_{i}\right)}$,

(ii) $U_{\left(\mu_{i}, v_{i}\right)} \sqsubset U_{\left(\mu_{i+1}, v_{i+1}\right)}$,

are satisfied, is illustrated as the fundamental sequence (FS) of $G$. The sequence is denoted by FS $(G)$.The $\left(\mu_{i}, v_{i}\right)$-level hypergraphs $\left\{G_{\left(\mu_{1}, v_{1}\right)}, G_{\left(\mu_{2}, v_{2}\right)}, \ldots, G_{\left(\mu_{n}, v_{n}\right)}\right\}$ are called the core hypergraphs of $G$. This is also called the core set of $G$ and is denoted by $c(G)$.

Definition 15. Let $G=(T, U)$ be a BF directed hypergraph and $F S(G)=\left\{\left(\mu_{1}, v_{1}\right),\left(\mu_{2}, v_{2}\right), \ldots,\left(\mu_{n}, v_{n}\right)\right\}$. If for each $E=\left(m^{+}, m^{-}\right) \in U$ and each $\mu_{i}, v_{i} \in F S(G), E_{(\mu, v)}=U_{\left(\mu_{i}, v_{i}\right)}$, for all $(\mu, v) \in\left(\left(\mu_{i-1, v_{i-1}}\right),\left(\mu_{i}, v_{i}\right)\right]$, then $G$ is sectionally elementary.

Definition 16. Let $G=(T, U)$ be a BF directed hypergraph and $c(G)=\left\{G_{\left(\mu_{1}, v_{1}\right)}, G_{\left(\mu_{2}, v_{2}\right)}, \ldots, G_{\left(\mu_{n}, v_{n}\right)}\right\}$. $G$ is said to be ordered if $c(G)$ is ordered. That is, $G_{\left(\mu_{1}, v_{1}\right)} \subset G_{\left(\mu_{2}, v_{2}\right)} \subset \ldots \subset G_{\left(\mu_{n}, v_{n}\right)}$. The BF directed hypergraph is called simply ordered if the sequence $\left\{G_{\left(\mu_{1}, v_{1}\right)}, G_{\left(\mu_{2}, v_{2}\right)}, \ldots, G_{\left(\mu_{n}, v_{n}\right)}\right\}$ is simply ordered.

Example 3. Consider a BF directed hypergraph $G=(T, U)$, such that $T=\left\{t_{1}, t_{2}, t_{3}, t_{4}, t_{5}\right\}$, $U=\left\{U_{1}, U_{2}, U_{3}\right\}$, given by the incidence matrix in Table 3. 
Table 3. BF directed hypergraph.

\begin{tabular}{cccc}
\hline $\boldsymbol{I}$ & $\boldsymbol{U}_{\mathbf{1}}$ & $\boldsymbol{U}_{\mathbf{2}}$ & $\boldsymbol{U}_{\mathbf{3}}$ \\
\hline$t_{1}$ & $\langle 0.8,-0.2\rangle$ & $\langle 0.6,-0.1\rangle$ & $\langle 0.4,-0.3\rangle$ \\
$t_{2}$ & $\mathbf{0}$ & $\mathbf{0}$ & $\langle 0.4,-0.3\rangle$ \\
$t_{3}$ & $\langle 0.8,-0.1\rangle$ & $\mathbf{0}$ & $\mathbf{0}$ \\
$t_{4}$ & $\langle 0.8,-0.2\rangle$ & $\langle 0.6,-0.1\rangle$ & $\langle 0.4,-0.3\rangle$ \\
$t_{5}$ & $\mathbf{0}$ & $\langle 0.5,-0.1\rangle$ & $\langle 0.4,-0.3\rangle$ \\
\hline
\end{tabular}

The corresponding graph is shown in Figure 3.

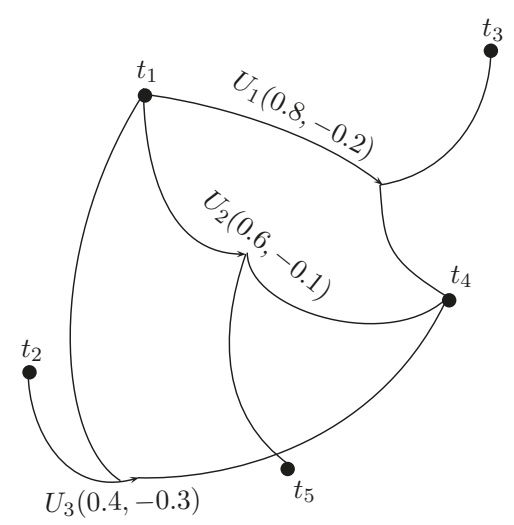

Figure 3. BF directed hypergraph.

By computing the $\left(\mu_{i}, v_{i}\right)$-level BF directed hypergraphs of $G$, we have $U_{(0.8,-0.2)}=\left\{t_{1}, t_{4}\right\}, U_{(0.6,-0.1)}=$ $\left\{t_{1}, t_{4}\right\}$ and $U_{(0.4,-0.3)}=\left\{\left\{t_{1}, t_{2}\right\},\left\{t_{4}, t_{5}\right\}\right\}$. Note that $G_{(0.8,-0.2)}=G_{(0.6,-0.1)}$ and $G_{(0.8,-0.2)} \subseteq G_{(0.4,-0.3)}$. The fundamental sequence is $F S(G)=\{(0.8,-0.2),(0.4,-0.3)\}$. The $(0.6,-0.1)$-level is not in FS $(G)$. Furthermore, $G_{(0.8,-0.2)} \neq G_{(0.4,-0.3)}$. G is not sectionally elementary since $U_{2(\mu, v)} \neq U_{2(0.8,-0.2)}$ for $\mu=0.6$, $v=-0.1$. The BF directed hypergraph is ordered, and the set of core hypergraphs is $c(G)=\left\{G_{1}=\right.$ $\left.G_{(0.8,-0.2)}, G_{2}=G_{(0.4,-0.3)}\right\}$. The induced fundamental sequence of $G$ is given in Figure 4.

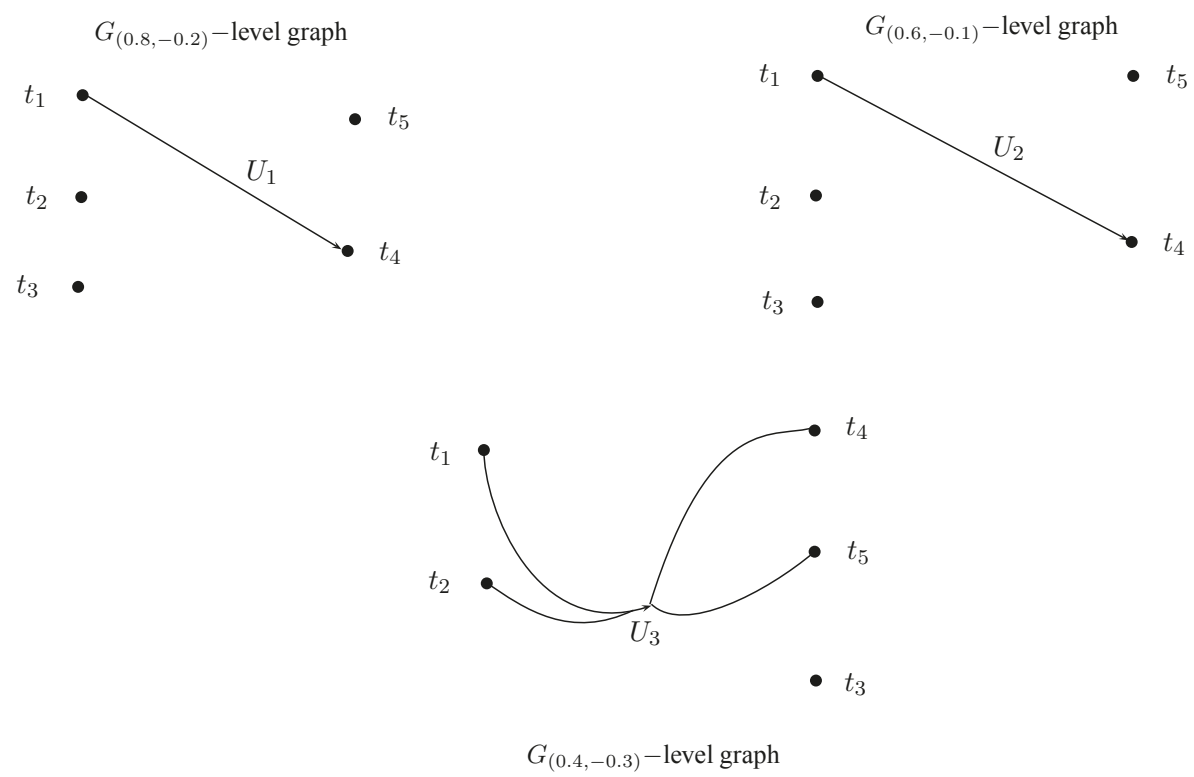

Figure 4. $G$ induced fundamental sequence. 


\section{Theorem 2.}

(a) If $G=(T, U)$ is an elementary $B F$ directed hypergraph, then $G$ is ordered.

(b) If $G$ is an ordered BF directed hypergraph with $c(G)=\left\{G_{\left(\mu_{1}, v_{1}\right)}, G_{\left(\mu_{2}, v_{2}\right), \ldots, G_{\left(\mu_{n}, v_{n}\right)}}\right\}$ and if $G_{\left(\mu_{n}, v_{n}\right)}$ is simple, then $G$ is elementary.

We now define the index matrix representation and certain operations on BF directed hypergraphs.

Definition 17. Let $G=(T, U)$ be a BF directed hypergraph. Then, the index matrix of $G$ is of the form $[T, U \subset T \times T]$ as given in Table 4 , where $T=\left\{t_{1}, t_{2}, t_{3}, \cdots, t_{k}\right\}$ and $U=\left\langle m_{i j}^{+}, m_{i j}^{-}\right\rangle=$

Table 4. Index matrix representation of BF directed hypergraphs.

\begin{tabular}{ccccc}
\hline & $\boldsymbol{t}_{\mathbf{1}}$ & $\boldsymbol{t}_{\mathbf{2}}$ & $\cdots$ & $\boldsymbol{t}_{\boldsymbol{k}}$ \\
\hline$t_{1}$ & $\left\langle m_{11}^{+}, m_{11}^{-}\right\rangle$ & $\left\langle m_{12}^{+}, m_{12}^{-}\right\rangle$ & $\cdots$ & $\left\langle m_{1 k}^{+}, m_{1 k}^{-}\right\rangle$ \\
$t_{2}$ & $\left\langle m_{21}^{+}, m_{21}^{-}\right\rangle$ & $\left\langle m_{22}^{+}, m_{22}^{-}\right\rangle$ & $\cdots$ & $\left\langle m_{2 k}^{+}, m_{2 k}^{-}\right\rangle$ \\
$\vdots$ & $\vdots$ & $\vdots$ & $\vdots$ & $\vdots$ \\
$t_{k}$ & $\left\langle m_{k 1}^{+}, m_{k 1}^{-}\right\rangle$ & $\left\langle m_{k 2}^{+}, m_{k 2}^{-}\right\rangle$ & $\cdots$ & $\left\langle m_{k k}^{+}, m_{k k}^{-}\right\rangle$ \\
\hline
\end{tabular}

where $m_{i j}^{+} \in[0,1]$ and $m_{i j}^{-} \in[-1,0], \mathrm{i}, \mathrm{j}=1,2,3, \ldots, k$. The edge between two vertices $v_{i}$ and $v_{j}$ is indexed by $\left\langle m_{i j}^{+}, m_{i j}^{-}\right\rangle$, whose values can be find out by using the Cartesian products defined below.

Definition 18. Let $E$ be a fixed set of points. The Cartesian product of two BF sets $B_{1}$ and $B_{2}$ over $E$ is defined as:

i. $\quad B_{1} \times{ }_{1} B_{2}=\left\{\left\langle\left(v_{1}, v_{2}\right), \min \left(m^{+}\left(v_{1}\right), m^{+}\left(v_{2}\right)\right), \max \left(m^{-}\left(v_{1}\right), m^{-}\left(v_{2}\right)\right)\right\rangle \mid v_{1} \in B_{1}, v_{2} \in B_{2}\right\}$.

ii. $B_{1} \times{ }_{2} B_{2}=\left\{\left\langle\left(v_{1}, v_{2}\right), \max \left(m^{+}\left(v_{1}\right), m^{+}\left(v_{2}\right)\right), \min \left(m^{-}\left(v_{1}\right), m^{-}\left(v_{2}\right)\right)\right\rangle \mid v_{1} \in B_{1}, v_{2} \in B_{2}\right\}$.

Note that the Cartesian product $B_{1} \times_{i} B_{2}$ is a BF set, where $i=1,2$.

We now define some operations on BF directed hypergraphs.

Definition 19. The addition of BF directed hypergraphs $G_{1}=\left(T_{1}, U_{1},\left\langle m_{i}^{+}, m_{i}^{-}\right\rangle,\left\langle m_{i j}^{+}, m_{i j}^{-}\right\rangle\right)$and $G_{2}=\left(T_{2}, U_{2},\left\langle m_{p}^{+}, m_{p}^{-}\right\rangle,\left\langle m_{p q}^{+}, m_{p q}^{-}\right\rangle\right)$, which is denoted by $G=G_{1} \boxplus G_{2}$, is defined as $G_{1} \boxplus G_{2}=$ $\left[T_{1} \cup T_{2},\left\langle m_{r}^{+}, m_{r}^{-}\right\rangle,\left\langle m_{r s}^{+}, m_{r s}^{-}\right\rangle\right]$, where:

$$
\left\langle m_{r}^{+}, m_{r}^{-}\right\rangle= \begin{cases}\left\langle m_{i}^{+}, m_{i}^{-}\right\rangle, & \text {if } v_{r} \in T_{1}-T_{2}, \\ \left\langle m_{p}^{+}, m_{p}^{-}\right\rangle, & \text {if } v_{r} \in T_{2}-T_{1}, \\ \left\langle\max \left(m_{i}^{+}, m_{p}^{+}\right), \min \left(m_{i}^{-}, m_{p}^{-}\right)\right\rangle, & \text {if } v_{r} \in T_{1} \cap T_{2}, \\ \mathbf{0}, & \text { otherwise. }\end{cases}
$$

and:

$$
\left\langle m_{r s}^{+}, m_{r s}^{-}\right\rangle= \begin{cases}\left\langle m_{i j}^{+}, m_{i j}^{-}\right\rangle, & \text {if } v_{r}=v_{i} \in T_{1} \text { and } v_{s}=v_{j} \in T_{1}-T_{2}, \\ \left\langle m_{p q}^{+}, m_{p q}^{-}\right\rangle, & \text {or } v_{r}=v_{i} \in T_{1}-T_{2} \text { and } v_{s}=v_{j} \in T_{1}, \\ & \text { if } v_{r}=v_{p} \in T_{2} \text { and } v_{s}=v_{q} \in T_{2}-T_{1}, \\ \left\langle\max \left(m_{i j}^{+}, m_{p q}^{+}\right), \min \left(m_{i j}^{-}, m_{p q}^{-}\right)\right\rangle, & \text {if } v_{r}=v_{p} \in v_{2}-v_{1} \text { and } v_{s}=v_{q} \in T_{2}, T_{2} \text { and } \\ & v_{s}=v_{j}=v_{q} \in T_{2} \cap T_{1}, \\ 0, & \text { otherwise. }\end{cases}
$$


Example 4. Consider the BF directed hypergraphs $G_{1}=\left(T_{1}, U_{1}\right)$ and $G_{2}=\left(T_{2}, U_{2}\right)$, where $T_{1}=$ $\left\{t_{1}, t_{2}, t_{3}, \ldots, t_{8}\right\}, U_{1}=\left\{\left(\left\{t_{1}\right\}, t_{3}\right),\left(\left\{t_{1}, t_{2}\right\}, t_{4}\right),\left(\left\{t_{3}\right\}, t_{5}\right),\left(\left\{t_{1}, t_{5}\right\}, t_{6}\right),\left(\left\{t_{5}, t_{7}\right\}, t_{8}\right)\right\}$ and $T_{2}=\left\{t_{1}, t_{2}, \ldots, t_{5}\right\}$, $U_{2}=\left\{\left(\left\{t_{1}, t_{3}\right\}, t_{2}\right),\left(\left\{t_{2}\right\}, t_{2}\right),\left(\left\{t_{4}\right\}, t_{2}\right),\left(\left\{t_{3}, t_{5}\right\}, t_{4}\right),\left(\left\{t_{3}\right\}, t_{5}\right)\right\}$ as shown in Figures 5 and 6 , respectively.

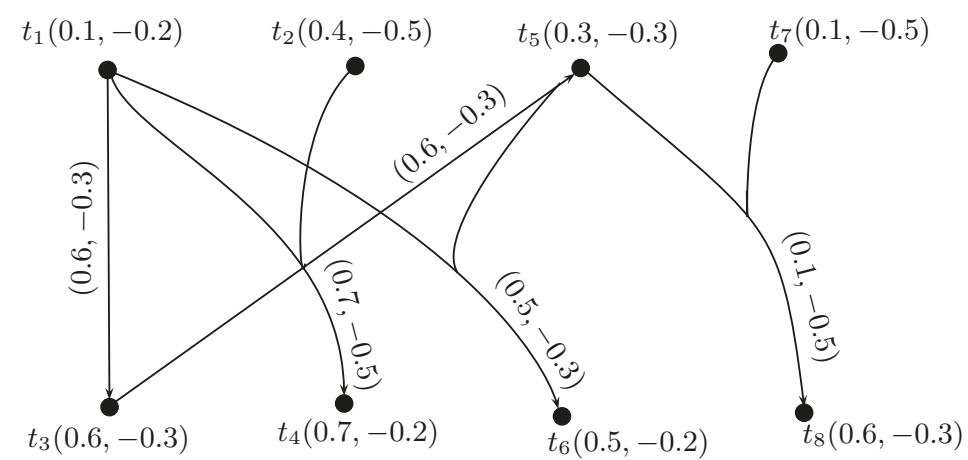

Figure 5. $G_{1}$.

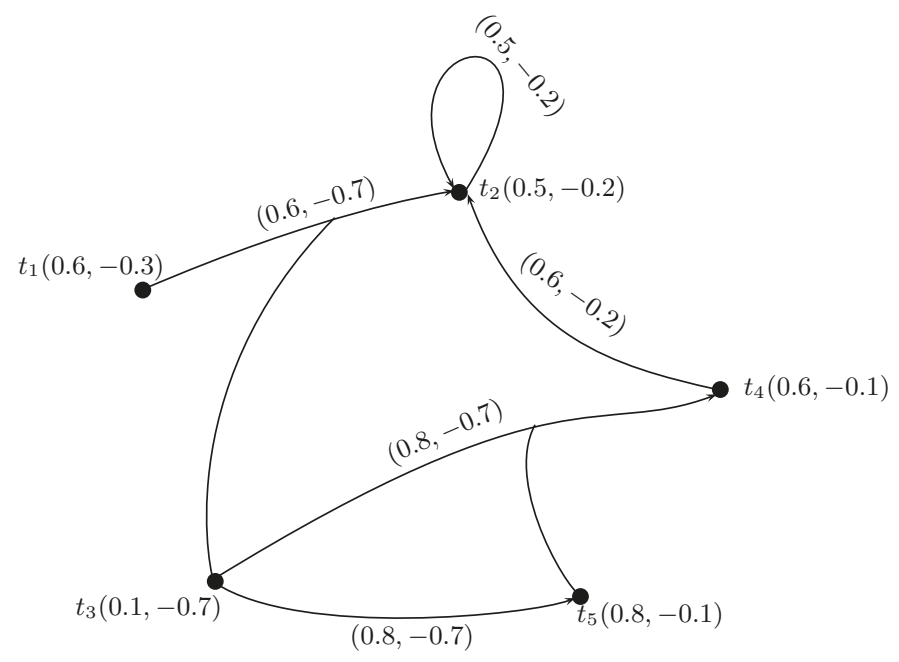

Figure 6. $G_{2}$.

The index matrix of $G_{1}$ is $\left[T_{1},\left\langle m_{i j}^{+}, m_{i j}^{-}\right\rangle\right]$as given in Table 5 , where $T_{1}=\left\{t_{1}, t_{2}, t_{3}, \ldots, t_{8}\right\}$ and:

Table 5. Index matrix of $G_{1}$.

\begin{tabular}{cccccccccc}
\hline & $\boldsymbol{t}_{\mathbf{1}}$ & $\boldsymbol{t}_{\mathbf{2}}$ & $\boldsymbol{t}_{\mathbf{3}}$ & $\boldsymbol{t}_{\mathbf{4}}$ & $\boldsymbol{t}_{\mathbf{5}}$ & $\boldsymbol{t}_{\mathbf{6}}$ & $\boldsymbol{t}_{\mathbf{7}}$ & $\boldsymbol{t}_{\mathbf{8}}$ \\
\cline { 2 - 8 } & $t_{1}$ & $\mathbf{0}$ & $\mathbf{0}$ & $\mathbf{0}$ & $\mathbf{0}$ & $\mathbf{0}$ & $\mathbf{0}$ & $\mathbf{0}$ & $\mathbf{0}$ \\
& $t_{2}$ & $\mathbf{0}$ & $\mathbf{0}$ & $\mathbf{0}$ & $\mathbf{0}$ & $\mathbf{0}$ & $\mathbf{0}$ & $\mathbf{0}$ & $\mathbf{0}$ \\
$\left\langle m_{i j}^{+}, m_{i j}^{-}\right\rangle=$ & $t_{3}$ & $\langle 0.6,-0.3\rangle$ & $\mathbf{0}$ & $\mathbf{0}$ & $\mathbf{0}$ & $\mathbf{0}$ & $\mathbf{0}$ & $\mathbf{0}$ & $\mathbf{0}$ \\
& $t_{4}$ & $\langle 0.7,-0.5\rangle$ & $\langle 0.7,-0.5\rangle$ & $\mathbf{0}$ & $\mathbf{0}$ & $\mathbf{0}$ & $\mathbf{0}$ & $\mathbf{0}$ & $\mathbf{0}$ \\
& $t_{5}$ & $\mathbf{0}$ & $\mathbf{0}$ & $\langle 0.6,-0.3\rangle$ & $\mathbf{0}$ & $\mathbf{0}$ & $\mathbf{0}$ & $\mathbf{0}$ & $\mathbf{0}$ \\
& $t_{6}$ & $\langle 0.5,-0.3\rangle$ & $\mathbf{0}$ & $\mathbf{0}$ & $\mathbf{0}$ & $\langle 0.5,-0.3\rangle$ & $\mathbf{0}$ & $\mathbf{0}$ & $\mathbf{0}$ \\
$t_{7}$ & $\mathbf{0}$ & $\mathbf{0}$ & $\mathbf{0}$ & $\mathbf{0}$ & $\mathbf{0}$ & $\mathbf{0}$ & $\mathbf{0}$ & $\mathbf{0}$ \\
$t_{8}$ & $\mathbf{0}$ & $\mathbf{0}$ & $\mathbf{0}$ & $\mathbf{0}$ & $\langle 0.1,-0.5\rangle$ & $\mathbf{0}$ & $\langle 0.1,-0.5\rangle$ & $\mathbf{0}$ \\
\hline
\end{tabular}

The index matrix of $G_{2}$ is $\left[T_{2},\left\langle m_{p q}^{+}, m_{p q}^{-}\right\rangle\right]$as given in Table 6 , where $T_{2}=\left\{t_{1}, t_{2}, t_{3}, t_{4}, t_{5}\right\}$ and: 
Table 6. Index matrix of $G_{2}$.

\begin{tabular}{ccccccc}
\hline & $\boldsymbol{t}_{\mathbf{1}}$ & $\boldsymbol{t}_{\mathbf{2}}$ & $\boldsymbol{t}_{\mathbf{3}}$ & $\boldsymbol{t}_{\mathbf{4}}$ & $\boldsymbol{t}_{\mathbf{5}}$ \\
\cline { 2 - 7 }$\left\langle m_{p q}^{+}, m_{p q}^{-}\right\rangle=$ & $t_{1}$ & $\mathbf{0}$ & $\mathbf{0}$ & $\mathbf{0}$ & $\mathbf{0}$ & $\mathbf{0}$ \\
& $t_{2}$ & $\langle 0.6,-0.7\rangle$ & $\langle 0.5,-0.2\rangle$ & $\langle 0.6,-0.7\rangle$ & $\langle 0.6,-0.2\rangle$ & $\mathbf{0}$ \\
$t_{3}$ & $\mathbf{0}$ & $\mathbf{0}$ & $\mathbf{0}$ & $\mathbf{0}$ & $\mathbf{0}$ \\
$t_{4}$ & $\mathbf{0}$ & $\mathbf{0}$ & $\langle 0.8,-0.7\rangle$ & $\mathbf{0}$ & $\langle 0.8,-0.7\rangle$ \\
$t_{5}$ & $\mathbf{0}$ & $\mathbf{0}$ & $\langle 0.8,-0.7\rangle$ & $\mathbf{0}$ & $\mathbf{0}$ \\
\hline
\end{tabular}

The index matrix of $G_{1} \boxplus G_{2}$ is $\left[T_{1} \cup T_{2},\left\langle m_{r s}^{+}, m_{r s}^{-}\right\rangle,\left\langle m_{r s}^{+}, m_{r s}^{-}\right\rangle\right]$, where $T_{1} \cup T_{2}=\left\{t_{1}, t_{2}, t_{3}, t_{4}, t_{5}, t_{6}, t_{7}, t_{8}\right\}$. The membership values $\left\langle m_{r}^{+}, m_{r}^{-}\right\rangle$are calculated by using Equation (1), and $\left\langle m_{r s}^{+}, m_{r s}^{-}\right\rangle$are calculated by using Equation (2) and are given in Table 7.

Table 7. Index matrix of $G_{1} \boxplus G_{2}$.

\begin{tabular}{rccccccccc}
\hline & $\boldsymbol{t}_{\mathbf{1}}$ & $\boldsymbol{t}_{\mathbf{2}}$ & $\boldsymbol{t}_{\mathbf{3}}$ & $\boldsymbol{t}_{\mathbf{4}}$ & $\boldsymbol{t}_{\mathbf{5}}$ & $\boldsymbol{t}_{\mathbf{6}}$ & $\boldsymbol{t}_{\mathbf{7}}$ & $\boldsymbol{t}_{\mathbf{8}}$ \\
\cline { 2 - 8 } & $t_{1}$ & $\mathbf{0}$ & $\mathbf{0}$ & $\mathbf{0}$ & $\mathbf{0}$ & $\mathbf{0}$ & $\mathbf{0}$ & $\mathbf{0}$ & $\mathbf{0}$ \\
$t_{2}$ & $\langle 0.6,-0.7\rangle$ & $\langle 0.5,-0.2\rangle$ & $\langle 0.6,-0.7\rangle$ & $\langle 0.6,-0.2\rangle$ & $\mathbf{0}$ & $\mathbf{0}$ & $\mathbf{0}$ & $\mathbf{0}$ \\
$\left\langle m_{r s}^{+}, m_{r s}^{-}\right\rangle=t_{3}$ & $\langle 0.6,-0.3\rangle$ & $\mathbf{0}$ & $\mathbf{0}$ & $\mathbf{0}$ & $\mathbf{0}$ & $\mathbf{0}$ & $\mathbf{0}$ & $\mathbf{0}$ \\
$t_{4}$ & $\langle 0.7,-0.5\rangle$ & $\langle 0.7,-0.5\rangle$ & $\langle 0.8,-0.7\rangle$ & $\mathbf{0}$ & $\langle 0.8,-0.7\rangle$ & $\mathbf{0}$ & $\mathbf{0}$ & $\mathbf{0}$ \\
$t_{5}$ & $\mathbf{0}$ & $\mathbf{0}$ & $\langle 0.8,-0.7\rangle$ & $\mathbf{0}$ & $\mathbf{0}$ & $\mathbf{0}$ & $\mathbf{0}$ & $\mathbf{0}$ \\
$t_{6}$ & $\langle 0.5,-0.3\rangle$ & $\mathbf{0}$ & $\mathbf{0}$ & $\mathbf{0}$ & $\langle 0.5,-0.3\rangle$ & $\mathbf{0}$ & $\mathbf{0}$ & $\mathbf{0}$ \\
$t_{7}$ & $\mathbf{0}$ & $\mathbf{0}$ & $\mathbf{0}$ & $\mathbf{0}$ & $\mathbf{0}$ & $\mathbf{0}$ & $\mathbf{0}$ & $\mathbf{0}$ \\
$t_{8}$ & $\mathbf{0}$ & $\mathbf{0}$ & $\mathbf{0}$ & $\mathbf{0}$ & $\langle 0.1,-0.5\rangle$ & $\mathbf{0}$ & $\langle 0.1,-0.5\rangle$ & $\mathbf{0}$ \\
\hline
\end{tabular}

The graph of $G_{1} \boxplus G_{2}$ is shown in Figure 7.

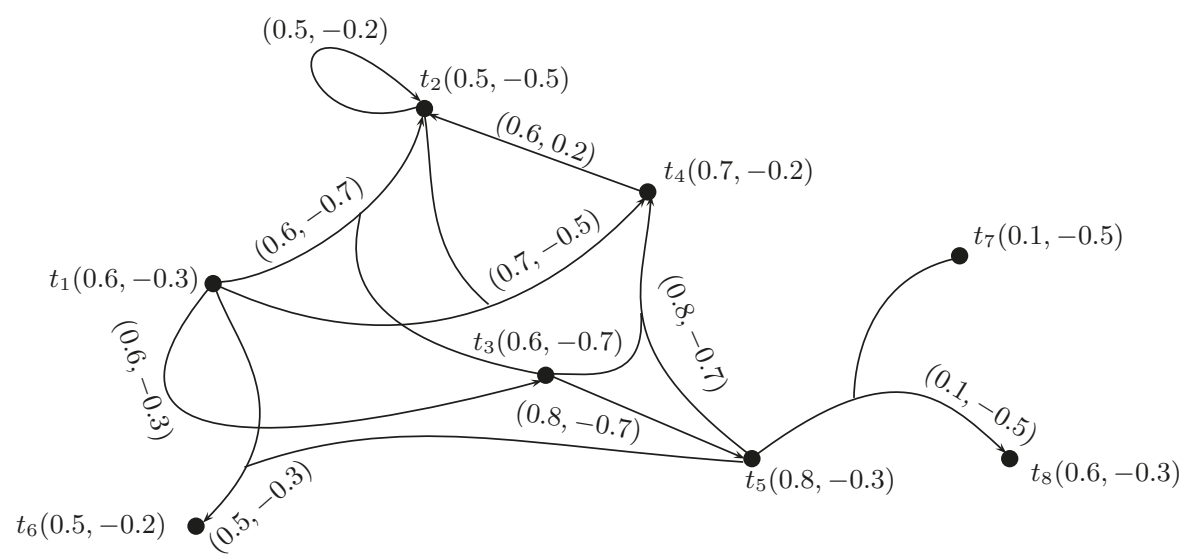

Figure 7. $G_{1} \boxplus G_{2}$.

Definition 20. The vertex-wise multiplication of two BF directed hypergraphs $G_{1}$ and $G_{2}$, denoted by $G_{1} \otimes G_{2}$, is $\left[T_{1} \cap T_{2},\left\langle m_{r}^{+}, m_{r}^{-}\right\rangle,\left\langle m_{r s}^{+}, m_{r s}^{-}\right\rangle\right]$, where:

$$
\begin{gathered}
\left\langle m_{r}^{+}, m_{r}^{-}\right\rangle=\left\langle\min \left(m_{i}^{+}, m_{p}^{+}\right), \max \left(m_{i}^{-}, m_{p}^{-}\right)\right\rangle \text {if } v_{r} \in T_{1} \cap T_{2}, \\
\left\langle m_{r s}^{+}, m_{r s}^{-}\right\rangle=\left\langle\min \left(m_{i j}^{+}, m_{p q}^{+}\right), \max \left(m_{i j}^{-}, m_{p q}^{-}\right)\right\rangle \text {if } v_{r}=v_{i}=v_{p} \in T_{1} \cap T_{2} \text { and } v_{s}=v_{j}=v_{q} \in T_{1} \cap T_{2} .
\end{gathered}
$$

Example 5. Consider BF directed hypergraphs $G_{1}$ and $G_{2}$ as shown in Figures 5 and 6, respectively. The index matrix of $G_{1} \otimes G_{2}$ is $\left[T_{1} \cap T_{2},\left\langle m_{r}^{+}, m_{r}^{-}\right\rangle,\left\langle m_{r s}^{+}, m_{r s}^{-}\right\rangle\right]$, where $T_{1} \cap T_{2}=\left\{t_{1}, t_{2}, t_{3}, t_{4}, t_{5}\right\}$. The membership values $\left\langle m_{r}^{+}, m_{r}^{-}\right\rangle$are calculated by using Equation (3), and $\left\langle m_{r s}^{+}, m_{r s}^{-}\right\rangle$are calculated by using Equation (4) and are given in Table 8. 
Table 8. Index matrix of $G_{1} \otimes G_{2}$.

\begin{tabular}{ccccccc}
\hline & $\boldsymbol{t}_{\mathbf{1}}$ & $\boldsymbol{t}_{\mathbf{2}}$ & $\boldsymbol{t}_{\mathbf{3}}$ & $\boldsymbol{t}_{\mathbf{4}}$ & $\boldsymbol{t}_{\mathbf{5}}$ \\
\cline { 2 - 6 } & $t_{1}$ & $\mathbf{0}$ & $\mathbf{0}$ & $\mathbf{0}$ & $\mathbf{0}$ & $\mathbf{0}$ \\
$\left.t_{r s}^{+}, m_{r s}^{-}\right\rangle=t_{2}$ & $\mathbf{0}$ & $\mathbf{0}$ & $\mathbf{0}$ & $\mathbf{0}$ & $\mathbf{0}$ \\
$t_{3}$ & $\mathbf{0}$ & $\mathbf{0}$ & $\mathbf{0}$ & $\mathbf{0}$ & $\mathbf{0}$ \\
$t_{4}$ & $\mathbf{0}$ & $\mathbf{0}$ & $\mathbf{0}$ & $\mathbf{0}$ & $\mathbf{0}$ \\
$t_{5}$ & $\mathbf{0}$ & $\mathbf{0}$ & $\langle 0.6,-0.3\rangle$ & $\mathbf{0}$ & $\mathbf{0}$ \\
\hline
\end{tabular}

The graph of $G_{1} \otimes G_{2}$ is given in Figure 8.

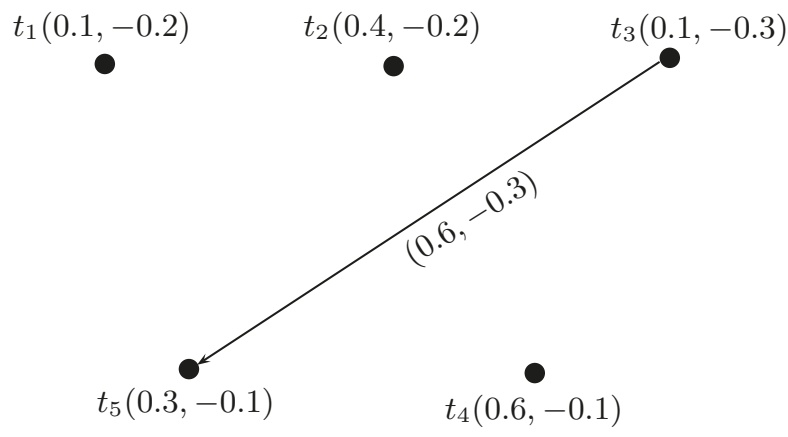

Figure 8. $G_{1} \otimes G_{2}$.

Definition 21. The multiplication of two BF directed hypergraphs $G_{1}$ and $G_{2}$, denoted by $G_{1} \odot G_{2}$, is defined as $\left[T_{1} \cup\left(T_{2}-T_{1}\right), T_{2} \cup\left(T_{1}-T_{2}\right),\left\langle m_{r}^{+}, m_{r}^{-}\right\rangle,\left\langle m_{r s}^{+}, m_{r s}^{-}\right\rangle\right]$, where:

$$
\left\langle m_{r}^{+}, m_{r}^{-}\right\rangle= \begin{cases}\left\langle m_{i}^{+}, m_{i}^{-}\right\rangle, & \text {if } v_{r} \in T_{1} \\ \left\langle m_{p}^{+}, m_{p}^{-}\right\rangle, & \text {if } \quad v_{r} \in T_{2} \\ \left\langle\min \left(m_{i}^{+}, m_{p}^{+}\right), \max \left(m_{i}^{-}, m_{p}^{-}\right)\right\rangle, & \text {if } \quad v_{r} \in T_{1} \cap T_{2}\end{cases}
$$

and:

$$
\left\langle m_{r s}^{+}, m_{r s}^{-}\right\rangle= \begin{cases}\left\langle m_{i j}^{+}, m_{i j}^{-}\right\rangle, & \text {if } v_{r}=v_{i} \in T_{1} \text { and } v_{s}=v_{j} \in T_{1}-T_{2}, \\ \left\langle m_{p q}^{+}, m_{p q}^{-}\right\rangle, & \text {if } v_{r}=v_{p} \in T_{2} \text { and } v_{s}=v_{q} \in T_{2}-T_{1}, \\ \left\langle\max \left(\min \left(m_{i j}^{+}, m_{p q}^{+}\right)\right), \min \left(\max \left(m_{i j}^{-}, m_{p q}^{-}\right)\right)\right\rangle, & \text {if } v_{r}=v_{i} \in T_{1} \cap T_{2} \\ 0, & \text { and } v_{s}=v_{q} \in T_{1} \cap T_{2}, \\ 0, & \text { otherwise. }\end{cases}
$$

Remark 1. The positive membership and negative membership values of the loops $\left(m_{r}^{+}, m_{r}^{-}\right)$in the resultant graph (if present) can be calculated as $m_{r}^{+} \leq m_{i}^{+}$or $m_{r}^{+} \leq m_{p}^{+}$and $m_{r}^{-} \geq m_{i}^{-}$or $m_{r}^{-} \geq m_{p}^{-}$.

Example 6. The index matrix of graph $G_{1} \odot G_{2}$ is $\left[T_{1} \cup\left(T_{2}-T_{1}\right), T_{2} \cup\left(T_{1}-T_{2}\right),\left\langle m_{r}^{+}, m_{r}^{-}\right\rangle,\left\langle m_{r s}^{+}, m_{r s}^{-}\right\rangle\right]$, where $T_{2} \cup\left(T_{1}-T_{2}\right)=\left\{t_{1}, t_{2}, t_{3}, \ldots, t_{8}\right\}$. The membership values $\left\langle m_{r}^{+}, m_{r}^{-}\right\rangle$are calculated by using Equation (5), and $\left\langle m_{r s}^{+}, m_{r s}^{-}\right\rangle$are calculated by using Equation (6) and are given in Table 9. 
Table 9. Index matrix of graph $G_{1} \odot G_{2}$.

\begin{tabular}{|c|c|c|c|c|c|c|c|c|c|}
\hline \multirow{9}{*}{$\left\langle m_{r s}^{+}, m_{r s}^{-}\right\rangle=$} & & $t_{1}$ & $t_{2}$ & $t_{3}$ & $t_{4}$ & $t_{5}$ & $t_{6}$ & $t_{7}$ & $t_{8}$ \\
\hline & $t_{1}$ & 0 & 0 & 0 & 0 & 0 & 0 & 0 & 0 \\
\hline & $t_{2}$ & 0 & 0 & 0 & 0 & 0 & 0 & 0 & 0 \\
\hline & $t_{3}$ & 0 & 0 & 0 & 0 & 0 & 0 & 0 & 0 \\
\hline & $t_{4}$ & $\langle 0.6,-0.5\rangle$ & $\langle 0.5,-0.2\rangle$ & $\langle 0.6,-0.5\rangle$ & $\langle 0.6,-0.2\rangle$ & 0 & 0 & 0 & 0 \\
\hline & $t_{5}$ & 0 & 0 & 0 & 0 & 0 & 0 & 0 & 0 \\
\hline & $t_{6}$ & $\langle 0.5,-0.3\rangle$ & 0 & 0 & 0 & $\langle 0.5,-0.3\rangle$ & 0 & 0 & 0 \\
\hline & $t_{7}$ & 0 & 0 & 0 & 0 & 0 & 0 & 0 & 0 \\
\hline & $t_{8}$ & 0 & 0 & 0 & 0 & $\langle 0.1,-0.5\rangle$ & 0 & $\langle 0.1,-0.5\rangle$ & 0 \\
\hline
\end{tabular}

The graph of $G_{1} \odot G_{2}$ is shown in Figure 9.

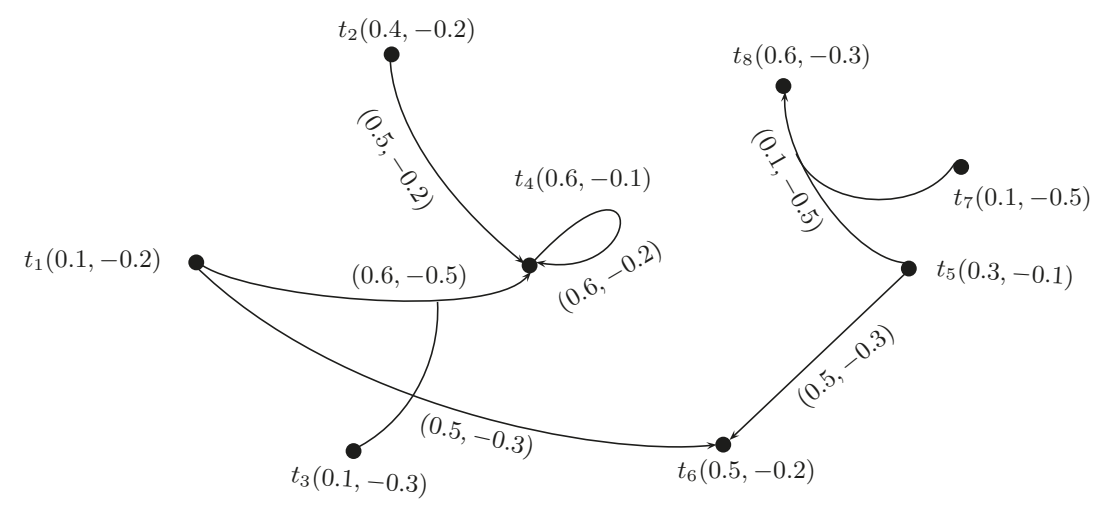

Figure 9. $G_{1} \odot G_{2}$.

Definition 22. The structural subtraction of $G_{1}$ and $G_{2}$, denoted by $G_{1} \boxminus G_{2}$, is defined as $\left[T_{1}-T_{2},\left\langle m_{r}^{+}, m_{r}^{-}\right\rangle,\left\langle m_{r s}^{+}, m_{r s}^{-}\right\rangle\right]$, where "-" is the set theoretic difference operation and:

$$
\begin{gathered}
\left\langle m_{r}^{+}, m_{r}^{-}\right\rangle= \begin{cases}\left\langle m_{i}^{+}, m_{i}^{-}\right\rangle, & \text {if } v_{r} \in T_{1}, \\
\left\langle m_{p}^{+}, m_{p}^{-}\right\rangle, & \text {if } v_{r} \in T_{2}, \\
0, & \text { otherwise. }\end{cases} \\
\left\langle m_{r s}^{+}, m_{r s}^{-}\right\rangle=\left\langle m_{i j}^{+}, m_{i j}^{-}\right\rangle, \text {if } v_{r}=v_{s} \in T_{1}-T_{2} \text { and } v_{s}=v_{j} \in T_{1}-T_{2} .
\end{gathered}
$$

If $T_{1}-T_{2}=\varnothing$, then the graph of $G_{1} \boxminus G_{2}$ is also empty.

Example 7. Consider BF directed hypergraphs $G_{1}$ and $G_{2}$ as shown in Figures 5 and 6 . The index matrix of $G_{1} \boxminus G_{2}$ is $\left[T_{1}-T_{2},\left\langle m_{r}^{+}, m_{r}^{-}\right\rangle,\left\langle m_{r s}^{+}, m_{r s}^{-}\right\rangle\right]$, where $T_{1}-T_{2}=\left\{t_{6}, t_{7}, t_{8}\right\}$. The membership values $\left\langle m_{r}^{+}, m_{r}^{-}\right\rangle$ are calculated by using Equation (7), and $\left\langle m_{r s}^{+}, m_{r s}^{-}\right\rangle$are calculated by using Equation (8) and are given in Table 10.

Table 10. Index matrix of $G_{1} \boxminus G_{2}$.

\begin{tabular}{ccccc}
\hline & & $\boldsymbol{t}_{\mathbf{6}}$ & $\boldsymbol{t}_{\mathbf{7}}$ & $\boldsymbol{t}_{\mathbf{8}}$ \\
\cline { 2 - 5 }$\left\langle m_{r s}^{+}, m_{r s}^{-}\right\rangle=$ & $t_{6}$ & $\mathbf{0}$ & $\mathbf{0}$ & $\mathbf{0}$ \\
& $t_{7}$ & $\mathbf{0}$ & $\mathbf{0}$ & $\mathbf{0}$ \\
& $t_{8}$ & $\mathbf{0}$ & $\langle 0.1,-0.5\rangle$ & $\mathbf{0}$ \\
\hline
\end{tabular}

The following Figure 10 shows their structural subtraction. 


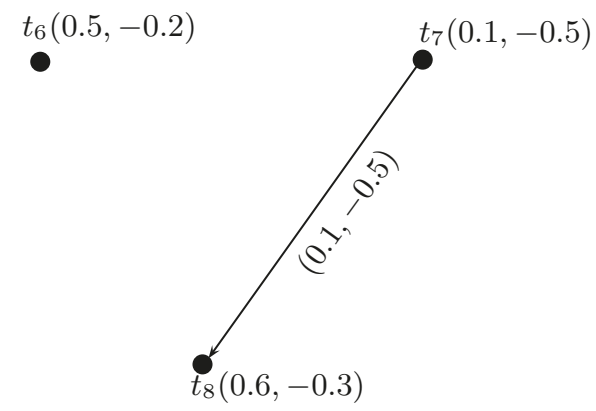

Figure 10. $G_{1} \boxminus G_{2}$.

Definition 23. $A$ BF directed hypergraph $G=(T, U)$ is called $B=\left(m^{+}, m^{-}\right)$-tempered $B F$ directed hypergraph of $G=(T, U)$ if there exists a crisp hypergraph $G^{*}=\left(T, U^{*}\right)$ and a $B F$ set $B=\left(m^{+}, m^{-}\right): \longrightarrow$ $[0,1] \times[-1,0]$, such that $U=\left\{F_{X}=\left(m^{+}, m^{-}\right) \mid X \in U^{*}\right\}$, where:

$$
m^{+}(x)=\left\{\begin{array}{ll}
\min \left(m^{+}(t) \mid t \in X\right), & \text { if } x \in X, \\
0, & \text { otherwise. }
\end{array} m^{-}(x)= \begin{cases}\max \left(m^{-}(t) \mid t \in X\right), & \text { if } x \in X, \\
0, & \text { otherwise }\end{cases}\right.
$$

Let $B \otimes G$ denotes the B-tempered hypergraph of $G$, which is firmed by the crisp hypergraph $G^{*}=\left(T, U^{*}\right)$ and the $B F$ set $B: T \longrightarrow[0,1] \times[-1,0]$.

Example 8. Consider the BF directed hypergraph $G=(T, U)$, where $T=\left\{t_{1}, t_{2}, t_{3}, t_{4}\right\}$ and $U=\left\{U_{1}, U_{2}, U_{3}\right\}$; the corresponding incidence matrix is given in Table 11 .

Table 11. Incidence matrix of $G$.

\begin{tabular}{cccc}
\hline & $\boldsymbol{U}_{\mathbf{1}}$ & $\boldsymbol{U}_{\mathbf{2}}$ & $\boldsymbol{U}_{\mathbf{3}}$ \\
\hline$t_{1}$ & $\langle 0.5,-0.2\rangle$ & $\langle 0.4,-0.3\rangle$ & $\mathbf{0}$ \\
$t_{2}$ & $\langle 0.5,-0.2\rangle$ & $\mathbf{0}$ & $\langle 0.5,-0.2\rangle$ \\
$t_{3}$ & $\langle 0.5,-0.2\rangle$ & $\mathbf{0}$ & $\langle 0.5,-0.2\rangle$ \\
$t_{4}$ & $\mathbf{0}$ & $\langle 0.4,-0.3\rangle$ & $\mathbf{0}$ \\
\hline
\end{tabular}

The corresponding graph is shown in Figure 11.

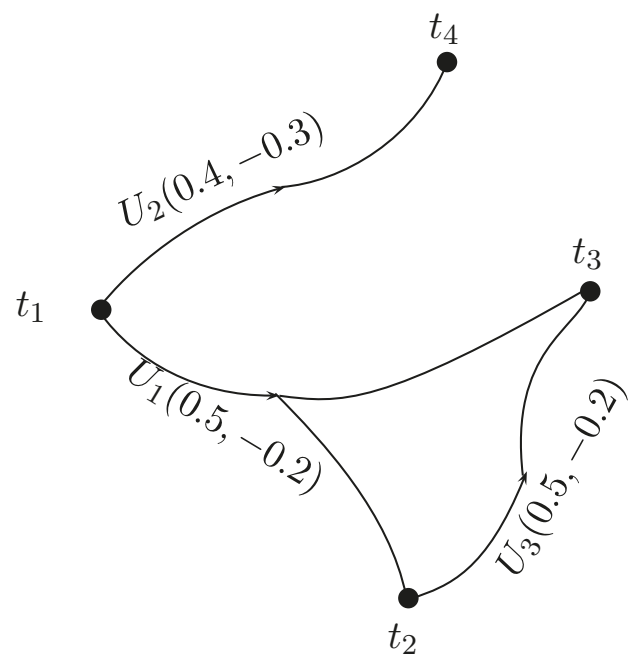

Figure 11. B-tempered BF directed hypergraph. 
Then, $U_{(0.5,-0.2)}=\left\{\left\{t_{1},\left\{t_{2}, t_{3}\right\}\right\},\left\{t_{2}, t_{3}\right\}\right\}, U_{(0.4,-0.3)}=\left\{\left\{t_{1}, t_{4}\right\}\right\}$.

Define $B=\left(m^{+}, m^{-}\right): T \longrightarrow[0,1] \times[-1,0]$ by $m^{+}\left(t_{1}\right)=0.6, m^{+}\left(t_{2}\right)=0.5, m^{+}\left(t_{3}\right)=0.5$, $m^{+}\left(t_{4}\right)=0.4, m^{-}\left(t_{1}\right)=-0.3, m^{-}\left(t_{2}\right)=-0.2, m^{-}\left(t_{3}\right)=-0.4, m^{-}\left(t_{4}\right)=-0.3$.

Note that:

$$
\begin{gathered}
m_{F\left\{t_{1}, t_{2}, t_{3}\right\}}^{+}=\min \left(m^{+}\left(t_{1}\right), m^{+}\left(t_{2}\right), m^{+}\left(t_{3}\right)\right)=0.5, \\
m_{F\left\{t_{1}, t_{2}, t_{3}\right\}}=\max \left(m^{-}\left(t_{1}\right), m^{-}\left(t_{2}\right), m^{-}\left(t_{3}\right)\right)=-0.2, \\
m_{F\left\{t_{1}, t_{4}\right\}}^{+}=\min \left(m^{+}\left(t_{1}\right), m^{+}\left(t_{4}\right)\right)=0.4, \\
m_{F\left\{t_{1}, t_{4}\right\}}^{-}=\max \left(m^{+}\left(t_{1}\right), m^{+}\left(t_{4}\right)\right)=-0.3, \\
m_{F\left\{t_{2}, t_{3}\right\}}^{+}=\min \left(m^{+}\left(t_{2}\right), m^{+}\left(t_{3}\right)\right)=0.5, \\
m_{F\left\{t_{2}, t_{3}\right\}}=\max \left(m^{+}\left(t_{2}\right), m^{+}\left(t_{3}\right)\right)=-0.2 .
\end{gathered}
$$

Thus:

$$
U_{1}=\left(m_{F\left\{t_{1}, t_{2}, t_{3}\right\}}^{+}, m_{F\left\{t_{1}, t_{2}, t_{3}\right\}}^{-}\right), U_{2}=\left(m_{F\left\{t_{1}, t_{4}\right\}}^{+}, m_{F\left\{t_{1}, t_{4}\right\}}^{-}\right), U_{3}=\left(m_{F\left\{t_{2}, t_{3}\right\}}^{+}, m_{F\left\{t_{2}, t_{3}\right\}}^{-}\right) .
$$

Hence, $G$ is a B-tempered BF directed hypergraph.

Theorem 3. A BF directed hypergraph $G=(T, U)$ is a $B=\left(m^{+}, m^{-}\right)$-tempered $B F$ directed hypergraph determined by some crisp hypergraph $G^{*}$ if and only if $G$ is elementary, simply ordered and support simple.

Proof. Suppose that $G=(T, U)$ is a B-tempered BF directed hypergraph, which is firmed by some crisp hypergraph $G^{*}$. Since $G$ is B-tempered, then the positive membership values and negative membership values of BF directed hyperedges are the same. Hence, $G$ is elementary. If the support of two BF directed hyperedges of the B-tempered BF directed hypergraph is the same, then the BF hyperedges are equal. Hence, $G$ is support simple. Let $c(G)=\left\{G_{\left(\mu_{1}, v_{1}\right)}, G_{\left(\mu_{2}, v_{2}\right)}, \ldots, G_{\left(\mu_{n}, v_{n}\right)}\right\}$. Since $G$ is elementary, it will be ordered.

Claim: $G$ is simply ordered.

Let $U \in G_{\mu_{i+1}, v_{i+1}}-G_{\mu_{i}, v_{i}}$, then there exists $v_{i} \in U$, such that $m_{i j}^{+}\left(v_{i}\right)=\mu_{i+1}$ and $m_{i j}^{-}\left(v_{i}\right)=v_{i+1}$. Since $\mu_{i+1}<\mu_{i}$ and $v_{i+1}<v_{i}$, it follows that $v_{i} \notin G_{\mu_{i}, v_{i}}$ and $U \nsubseteq G_{\mu_{i}, v_{i}}$. Hence, $G$ is simply ordered.

Conversely, suppose $G=(T, U)$ is elementary, simply ordered and support simple. As we know, $G_{\mu_{i}, v_{i}}=G_{i}=\left(T_{i}, U_{i}\right)$ and $m_{i j}^{+}: T \longrightarrow[0,1]$ and $m_{i j}^{-}: T \longrightarrow[-1,0]$ are defined by:

$$
\begin{aligned}
& m_{i j}^{+}=\left\{\begin{array}{ccc}
\mu_{1}, & \text { if } \quad v_{i} \in T_{1}, \\
\mu_{i}, & \text { if } & v_{i} \in T_{i}-T_{i-1}, i=1,2,3, \cdots, m .
\end{array}\right.
\end{aligned}
$$

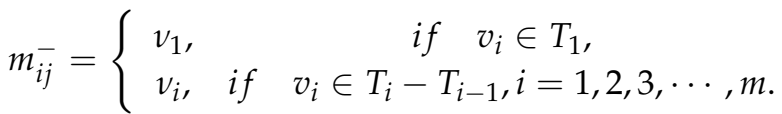

To prove $U=\left\{m_{i j}^{+}\left(v_{i}\right), m_{i j}^{-}\left(v_{i}\right) \mid v_{i} \in U_{i}\right\}$, where:

$$
m_{i j}^{+}\left(v_{i}\right)=\left\{\begin{array}{ll}
\min m_{i}^{+}(y) \mid y \in U, & \text { if } v_{i} \in U_{i}, \\
0, & \text { otherwise. }
\end{array} m_{i j}^{-}\left(v_{i}\right)= \begin{cases}\max m_{i}^{-}(y) \mid y \in U, & \text { if } v_{i} \in U_{i}, \\
0, & \text { otherwise. }\end{cases}\right.
$$

Let $U^{\prime} \in U_{i}$.

There is a unique BF hyperedge $\left(a_{i j}, b_{i j}\right)$ in $U$ having support $U^{\prime}$ because $G$ is elementary and support simple. Clearly, different edges in $U$ having distinct supports lie in $U_{i}$. We have to prove that for each $U^{\prime} \in U_{i}, m_{i j}^{+}\left(v_{i}\right)=a_{i j}, m_{i j}^{-}\left(v_{i}\right)=b_{i j}$. Since distinct edges have different supports and all edges are elementary, then the definition of the fundamental sequence implies that $h\left(a_{i j}, b_{i j}\right)$ is the same as an arbitrary element of $\left(\mu_{i}, v_{i}\right)$ of $F S(G)$. Therefore, $U^{\prime} \subseteq T_{i}$. Further, if $i>1$, then $U^{\prime} \in U_{i}-U_{i-1}$. Since $U \subseteq U_{i}$, the definition of B-tempered indicates that for each $v_{i} \in U_{i}, m_{i j}^{+}\left(v_{i}\right) \geq \mu_{i}$ and $m_{i j}^{-}\left(v_{i}\right) \leq v_{i}$. 
To prove $m_{i j}^{+}\left(v_{i}\right)=\mu_{i}$ and $m_{i j}^{-}\left(v_{i}\right)=v_{i}$ for some $V_{i} \in U_{i}$, it follows from the definition of $B$-tempered $m_{i j}^{+}\left(v_{i}\right) \geq \mu_{i-1}$ and $m_{i j}^{-}\left(v_{i}\right) \leq v_{i-1}$ for all $v_{i} \in U_{i} \Longrightarrow U \subseteq U_{i-1}$ and, so, $U \in U_{i}-U_{i-1}$. Since $G$ is simply ordered, therefore $U \nsubseteq U_{i-1}$, which is a contradiction to the definition of $B$-tempered BF directed hypergraphs. Thus, from the definition of $m_{i j}^{+}\left(v_{i}\right)$ and $m_{i j}^{-}\left(v_{i}\right)$, we have $m_{i j}^{+}=a_{i j}, m_{i j}^{-}=b_{i j}$.

Theorem 4. Let $G=(T, U)$ be a simply-ordered BF directed hypergraph and $F S(G)=\left\{\mu_{n}, \mu_{n-1}, \mu_{n-2}, \cdots\right.$, $\left.\mu_{1} v_{1}, v_{2}, \cdots, v_{n}\right\}$. If $G_{\mu_{n}, v_{n}}$ is a simple hypergraph, then there exists a partial BF directed hypergraph $D^{\prime}=\left(T, U^{\prime}\right)$ of $G$, such that the conditions given below are satisfied:

(i) $D^{\prime}$ is a B-tempered BF directed hypergraph of $G$.

(ii) $U \sqsubseteq U^{\prime}$, that is for all $\left(m^{+}, m^{-}\right) \in U$, there exist $\left(m^{+}, m^{-}\right) \in U^{\prime}$, such that $\left(m^{+}(U) \subseteq m^{+}\left(U^{\prime}\right)\right)$ and $\left(m^{-}(U) \subseteq m^{-}\left(U^{\prime}\right)\right)$.

(iii) $F S(G)=F S\left(D^{\prime}\right)$ and $c(G)=c\left(D^{\prime}\right)$.

Proof. By the above Theorem, we have $G$ is an elementary BF directed hypergraph. By the removal of all of those edges of $G$ which lie in another edge of $G$ properly, we attain the partial BF directed hypergraph $D^{\prime}=\left(T, U^{\prime}\right)$, where $U^{\prime}=\left\{U_{i} \in U \mid U_{i} \subseteq U_{j}\right.$ and $U_{j} \in U$, then $\left.U_{i}=U_{j}\right\}$. Since $G_{n_{n}, v_{n}}$ is simple and all of its edges are elementary, no edges can properly be contained in other edges of $G$ if they have different support. Hence, (iii) holds. We know that $D^{\prime}$ is support simple. Thus, all of the above conditions are satisfied by $D^{\prime}$. From the definition of $U^{\prime}, D^{\prime}$ is elementary and support simple. Thus, $D^{\prime}$ is B-tempered.

\section{Algorithm For Computing Minimum Arc Length and Shortest Hyperpath}

This section investigates the definition of the triangular BF number. The score and ranking of BF numbers are also defined. A triangular BF number is used to represent the arc length in a hypernetwork. The algorithm explained below is based on [16]. Let $L_{j}$ denotes the arc length of the $j$-th hyperpath.

Definition 24. Let $E$ be a finite set, which is non-empty, and $B=\left\langle m^{+}, m^{-}\right\rangle$be a BF set. Then, the pair $\left(m^{+}(a), m^{-}(a)\right)$ is called a BF number, denoted by $(\langle l, m, n\rangle,\langle c, d, e\rangle)$, where $\langle l, m, n\rangle \in F\left(I^{+}\right),\langle c, d, e\rangle \in$ $F\left(I^{-}\right), I^{+}=[0,1], I^{-}=[-1,0]$.

Definition 25. A triangular BF number $B$ is denoted by $B=\left\{\left\langle m^{+}(a), m^{-}(a)\right\rangle \mid a \in E\right\}$, where $m^{+}(a)$ and $m^{-}(a)$ are BF numbers. Therefore, a triangular $B F$ number is given by $\tilde{B}=(\langle l, m, n\rangle,\langle c, d, e\rangle)$. The diagrammatic representation of BF number $(\langle l, m, n\rangle,\langle c, d, e\rangle)$ is shown in Figure 12.

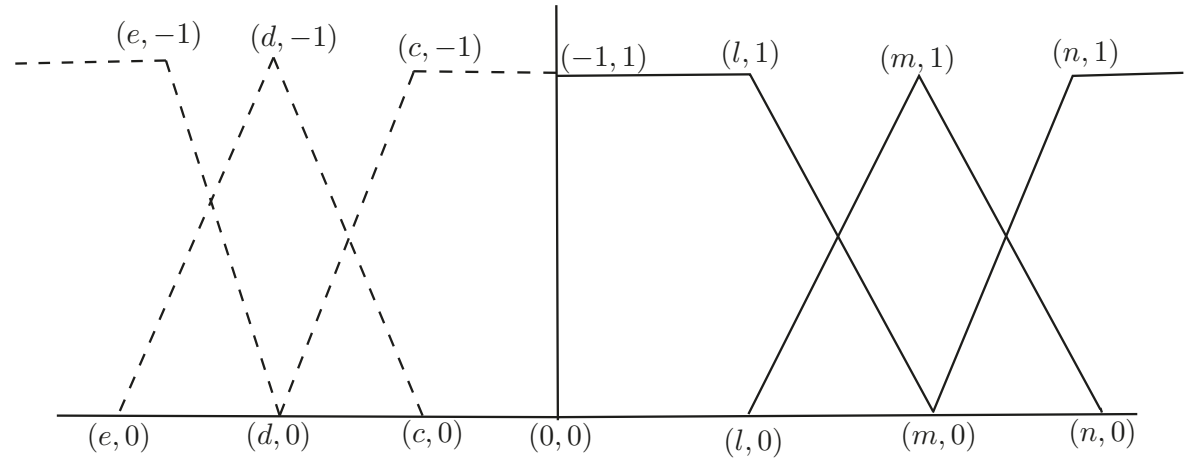

Figure 12. Triangular BF number.

Definition 26. Let $\tilde{B}=(\langle l, m, n\rangle,\langle c, d, e\rangle)$ be a triangular $B F$ number, then the score of $\tilde{B}$ is a BF set whose positive membership value is $S^{+}(\tilde{B})=\frac{l+2 m+n}{4}$ and negative membership value is $S^{-}(\tilde{B})=\frac{c+2 d+e}{4}$. 
Definition 27. The accuracy of a triangular BF number is defined as $\operatorname{Acc}(\tilde{B})=\frac{1}{2}\left(S^{+}(\tilde{B})+S^{-}(\tilde{B})\right)$.

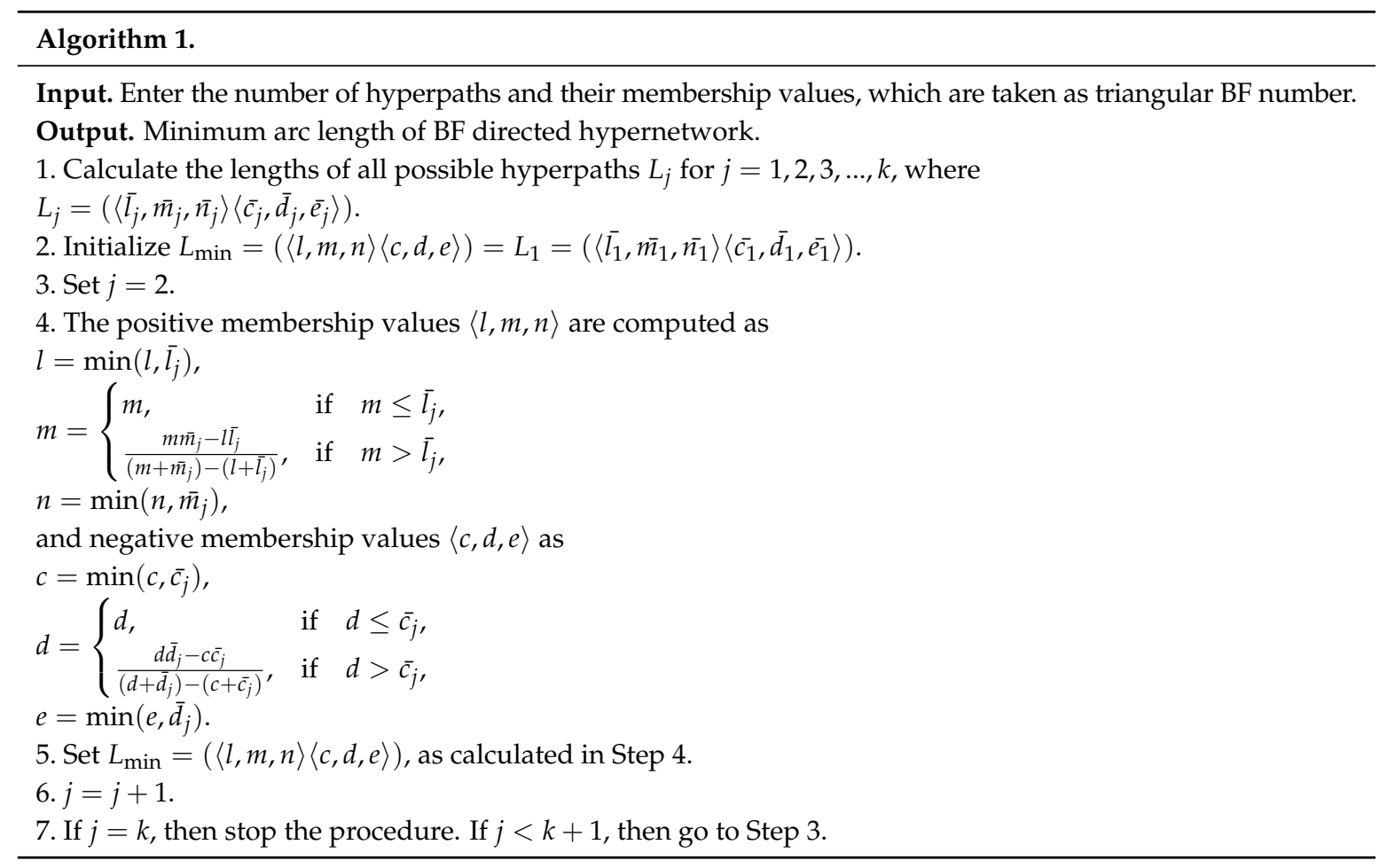

Example 9. Consider a hypernetwork with triangular BF arc lengths shown in Figure 13.

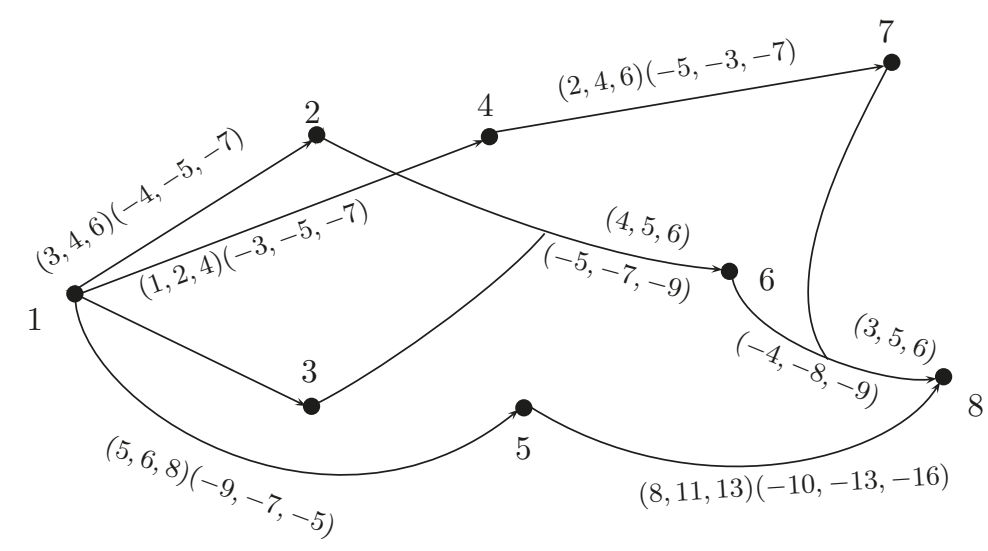

Figure 13. BF hypernetwork.

(1) From source Vertex 1 to destination Vertex 8, there are four possible paths $(k=4)$, given as follows:

Path(1): $1 \rightarrow 2 \rightarrow 6 \rightarrow 8, L_{1}=(\langle 10,14,18\rangle\langle-13,-20,-25\rangle)$;

Path(2): $1 \rightarrow 3 \rightarrow 6 \rightarrow 8, L_{2}=(\langle 12,16,20\rangle\langle-18,-22,-23\rangle)$;

Path(3): $1 \rightarrow 5 \rightarrow 8, L_{3}=(\langle 13,17,21\rangle\langle-19,-20,-21\rangle)$;

Path(4): $1 \rightarrow 4 \rightarrow 7 \rightarrow 8, L_{4}=(\langle 6,11,16\rangle\langle-12,-16,-23\rangle)$.

(2) Initialize $L_{\min }=(\langle l, m, n\rangle\langle c, d, e\rangle)=L_{1}=\left(\left\langle\bar{l}_{1}, \bar{m}_{1}, \bar{n}_{1}\right\rangle\left\langle\bar{c}_{1}, \bar{d}_{1}, \bar{e}_{1}\right\rangle\right)=(\langle 10,14,18\rangle\langle-13,-20,-25\rangle)$.

(3) Initialize $j=2$.

(4) Let $L_{\min }=(10,14,18)(-13,-20,-25)$ and $L_{2}=\left(\left\langle\overline{l_{2}}, \bar{m}_{2}, \overline{n_{2}}\right\rangle\left\langle\overline{c_{2}}, \overline{d_{2}}, \overline{c_{2}}\right\rangle\right)=$ $(12,16,20)(-18,-22,-23)$. Compute the positive membership values $\langle l, m, n\rangle$ as:

$$
l=\min \left(l, \overline{l_{2}}\right)=\min (10,12)=10,
$$




$$
\begin{aligned}
& m=\left\{\frac{(14 \times 16)-(10 \times 12)}{(14+16)-(10+12)}=13, \quad \text { since } m>\overline{l_{2}}\right. \text {, } \\
& n=\min \left(n, \bar{m}_{2}\right)=\min (18,16)=16,
\end{aligned}
$$

and negative membership values $\langle c, d, e\rangle$ as:

$$
\begin{gathered}
c=\min \left(c, \overline{c_{2}}\right)=\min (-13,-18)=-18, \\
d=\overline{d_{2}}=-22 \text { since } d<\overline{d_{2}}, \\
e=\min \left(e, \overline{d_{2}}\right)=\min (-25,-22)=-25 .
\end{gathered}
$$

(5) Set $L_{\min }=(\langle 10,13,16\rangle\langle-18,-22,-25\rangle)$.

(6) $j=j+1=3$.

(7) If $j<k+1$, go to Step 4 .

(4) Let $L_{\min }=(10,13,16)(-18,-22,-25)$ and $L_{3}=\left(\left\langle\overline{l_{3}}, \bar{m}_{3}, \overline{n_{3}}\right\rangle\left\langle\overline{c_{3}}, \overline{d_{3}}, \overline{e_{3}}\right\rangle\right)=$ $(13,17,21)(-19,-20,-21)$. Calculate the positive membership values as:

$$
\begin{gathered}
l=\min \left(l, \overline{l_{3}}\right)=\min (10,13)=10, \\
m=13 \text { since } m=\overline{l_{3}}, \\
n=\min \left(n, \bar{m}_{3}\right)=\min (16,17)=16,
\end{gathered}
$$

and negative membership values $\langle c, d, e\rangle$ as:

$$
\begin{gathered}
c=\min \left(c, \overline{c_{3}}\right)=\min (-18,-19)=-19, \\
d=-22 \operatorname{since} d<\overline{c_{3}}, \\
e=\min \left(e, \overline{d_{3}}\right)=\min (-25,-20)=-25,
\end{gathered}
$$

(5) Set $L_{\min }=(\langle 10,13,16\rangle\langle-19,-22,-25\rangle)$.

Repeat the procedure until $j=4$.

Finally, we get the minimum arc length of BF hypernetwork as:

$$
L_{\min }=(\langle 6,10.38,11\rangle\langle-19,-22,-25\rangle) .
$$

We now write steps of the score-based method to determine a BF shortest hyperpath.

(1) All possible hyperpaths are considered from the source point to the destination.

(2) Compute the scores of the hyperpaths.

(3) Find the accuracy of all paths.

(4) The shortest hyperpath is obtained with the lowest accuracy.

Example 10. Consider the BF hypernetwork as +shown in Figure 13. The BF shortest hyperpath in this hypernetwork is recognized using the score-based method. The scores of hyperpaths can be calculated a $6 \mathrm{~s}$ :

$$
\begin{gathered}
S\left(P_{1}\right)=\left(\frac{l+2 m+n}{4}, \frac{c+2 d+e}{4}\right)=\left(\frac{10+2(14)+18}{4}, \frac{-13+2(-20)-25}{4}\right)=(14,-19.5) . \\
S\left(P_{2}\right)=\left(\frac{12+2(16)+20}{4}, \frac{-18+2(-22)-23}{4}\right)=(16,-21.25) .
\end{gathered}
$$

Similarly, $S\left(P_{3}\right)=(17,-20)$ and $S\left(P_{4}\right)=(11,-16.75)$.

The accuracy of hyperpaths can be computed as:

$$
\begin{gathered}
\operatorname{Acc}\left(P_{1}\right)=\frac{1}{2}(14+(-19.5))=-2.75, A c c\left(P_{2}\right)=\frac{1}{2}(16+(-21.25))=-2.625, A c c\left(P_{3}\right) \\
=\frac{1}{2}(17+(-20)) \text { and } A c c\left(P_{4}\right)=\frac{1}{2}(11+(-16.75))=-2.875
\end{gathered}
$$

From Table 12 given below, the path $P_{4}: 1 \rightarrow 4 \rightarrow 7 \rightarrow 8$ with minimum accuracy is identified as the BF shortest hyperpath. 
Table 12. Accuracy table.

\begin{tabular}{cccc}
\hline Path & Score & Accuracy & Rank \\
\hline$P_{1}$ & $(14,-19.5)$ & -2.75 & 2 \\
$P_{2}$ & $(16,-21.25)$ & -2.625 & 3 \\
$P_{3}$ & $(17,-20)$ & -1.50 & 4 \\
$P_{4}$ & $(11,-16.75)$ & -2.875 & 1 \\
\hline
\end{tabular}

\section{Conclusions}

$\mathrm{BF}$ graph theory is widely applied in computer science and mathematics. In comparison to the classical and fuzzy models, BF models provide more precision, comparability and flexibility to the system as they combine the bipolarity and fuzziness both into a unified model. We have represented the $\mathrm{BF}$ directed hypergraphs using the incidence matrix and described their certain properties. We have represented the $\mathrm{BF}$ directed hypergraphs using the index matrix. We have described certain operations on $\mathrm{BF}$ directed hypergraphs, including addition, multiplication and structural subtraction. We have discussed an algorithm to find the minimum arc length of the BF directed hyperpath. We aim to widen our research work to: (1) BF soft directed hypergraphs; (2) bipolar neutrosophic hypergraphs; (3) rough neutrosophic graphs; and (4) soft rough directed hypergraphs.

Author Contributions: Both authors contributed equally to this manuscript.

Conflicts of Interest: The authors declare that there are no conflicts of interest regarding this manuscript.

\section{References}

1. Berge, C. Graphs and Hypergraphs; North-Holland Publishing Company: Amsterdam, The Netherlands, 1973.

2. Liu, D.R.; Wu, M.Y. A hypergraph based approach to declustering problems. Distrib. Parallel Databases 2001, 10, 269-288.

3. Ausiello, G.; Nanni, U.; Italiano, G.F. Dynamic maintenance of directed hypergraphs. Theor. Comput. Sci. 1990, 72, 97-117.

4. Gallo, G.; Longo, G.; Pallottin, S. Directed hypergraphs and applications. Discret. Appl. Math. 1993, 42, 177-201.

5. Zadeh, L.A. Fuzzy sets. Inf. Control 1965, 8, 338-353.

6. Zhang, W.-R. Bipolar fuzzy sets and relations: A computational framework forcognitive modeling and multiagent decision analysis. In Proceedings of the Fuzzy Information Processing Society Biannual Conference, San Antonio, TX, USA, 18-21 December 1994; pp. 305-309.

7. Kaufmann, A. Introduction a la Theorie des Sous-Ensembles Flous; Masson et Cie: Paris, France, 1973.

8. Zadeh, L.A. Similarity relations and fuzzy orderings. Inf. Sci. 1971, 3, 177-200.

9. Rosenfeld, A. Fuzzy graphs. In Fuzzy Sets and Their Applications; Zadeh, L.A., Fu, K.S., Shimura, M., Eds.; Academic Press: New York, NY, USA, 1975; pp. 77-95.

10. Bhattacharya, P. Some remarks on fuzzy graphs. Pattern Recogn. Lett. 1987, 6, 297-302.

11. Mordeson, J.N.; Chang-Shyh, P. Operations on fuzzy graphs. Inf. Sci. 1994, 79, 159-170.

12. Mordeson, J.N.; Nair, P.S. Fuzzy Graphs and Fuzzy Hypergraphs, 2nd ed.; Physica Verlag: Heidelberg, Germany, 2001.

13. Chen, S.M. Interval-valued fuzzy hypergraph and fuzzy partition. IEEE Trans. Syst. Man Cybern. Part B 1997, 27, 725-733.

14. Lee-Kwang, H.; Lee, K.-M. Fuzzy hypergraph and fuzzy partition. IEEE Trans. Syst. Man Cybern. 1995, 25, 196-201.

15. Parvathi, R.; Thilagavathi, S. Intuitionistic fuzzy directed hypergraphs. Adv. Fuzzy Sets Syst. 2013, 14, 39-52.

16. Rangasamy, P.; Akram, M.; Thilagavathi, S. Intuitionistic fuzzy shortest hyperpath in a network. Inf. Process. Lett. 2013, 113, 599-603.

17. Myithili, K.K.; Parvathi, R.; Akram, M. Certain types of intuitionistic fuzzy directed hypergraphs. Int. J. Mach. Learn. Cybern. 2016, 7, 287-295.

18. Akram, M. Bipolar fuzzy graphs. Inf. Sci. 2011, 181, 5548-5564. 
19. Akram, M.; Dudek, W.A. Regular bipolar fuzzy graphs. Neural Comput. Appl. 2012, 21, 197-205.

20. Akram, M.; Waseem, N. Novel applications of bipolar fuzzy graphs to decision making problems. J. Appl. Math. Comput. 2016, doi:10.1007/s12190-016-1062-3.

21. Sarwar, M.; Akram, M. Novel concepts of bipolar fuzzy competition graphs. J. Appl. Math. Comput. 2016, doi:10.1007/s12190-016-1021-z.

22. Samanta, S.; Pal, M. Bipolar fuzzy hypergraphs. Int. J. Fuzzy Log. Syst. 2012, 2, 17-28.

23. Akram, M.; Dudek, W.A.; Sarwar, S. Properties of bipolar fuzzy hypergraphs. Ital. J. Pure Appl. Math. 2013, 31, 141-160.

24. Han, Y.; Shi, P.; Chen, S. Bipolar-valued rough fuzzy set and its applications to decision information system. IEEE Trans. Fuzzy Syst. 2015, 23, 2358-2370.

25. Shi, P.; Su, X.; Li, F. Dissipativity-based filtering for fuzzy switched systems with stochastic perturbation. IEEE Trans. Autom. Control 2016, 61, 1694-1699.

26. Shi, P.; Zhang, Y.; Chadli, M.; Agarwal, R. Mixed H-infinity and passive filtering for discrete fuzzy neural networks with stochastic jumps and time delays. IEEE Trans. Neural Netw. Learn. Syst. 2016, 27, 903-909.

(C) 2017 by the authors. Licensee MDPI, Basel, Switzerland. This article is an open access article distributed under the terms and conditions of the Creative Commons Attribution (CC BY) license (http:/ / creativecommons.org/licenses/by/4.0/). 